\title{
Retraction Note: A model of economic loss of environmental carrying capacity caused by flood disasters in urban tourism areas
}

\author{
Bingwu Liao ${ }^{1} \cdot$ Songhao $\mathrm{Yu}^{1} \cdot$ Maoxuan Wang ${ }^{1}$
}

Published online: 1 December 2021

C) Saudi Society for Geosciences 2021

Retraction Note: Arabian Journal of Geosciences (2021) 14: 913 https://doi.org/10.1007/s12517-021-07223-y

The Editor-in-Chief and the Publisher have retracted this article because the content of this article is nonsensical. The peer review process was not carried out in accordance with the Publisher's peer review policy. Author Maoxuan Wang agrees with this retraction. Author Songhao Yu has not responded to correspondence regarding this retraction. The Publisher has not been able to obtain a current email address for author Bingwu Liao.

The original article can be found online at https://doi.org/10.1007/ s12517-021-07223-y.

Maoxuan Wang

202031260006@mail.bnu.edu.cn

1 School of Government, Beijing Normal University, Beijing 100875, China 